\title{
Development and Evaluation of a Mobile Search System for Science Experiments to Connect School Knowledge to Common Knowledge
}

\author{
Takahito Tomoto ${ }^{1}$, Tomoya Horiguchi ${ }^{2}$, and Tsukasa Hirashima ${ }^{3}$ \\ ${ }^{1}$ Faculty of Engineering, Tokyo University of Science 1-3 Kagurazaka, Shinjuku-ku, \\ Tokyo 162-8601 Japan \\ ${ }^{2}$ Graduate School of Maritime Sciences, Kobe University, 5-1-1 Fukaeminami-machi, \\ Higashinada-ku, Kobe-shi, Hyogo 658-0022, Japan \\ ${ }^{3}$ Graduate School of Engineering, Hiroshima University, 1-4-1 Kagamiyama, \\ Higashi Hiroshima City, Hiroshima 739-8527, Japan \\ tomoto@ms.kagu.tus.ac.jp
}

\begin{abstract}
In this paper, we propose a method that connects school knowledge to common knowledge through a mobile search system that enables users to think about and perform science experiments relevant to their everyday life. We developed the system and tested it in an evaluation experiment with 15 participants who used the system in everyday life over the course of a week. The evaluation results revealed that the users began to consider appropriate experiments, describe appropriate locations, and understand scientific concepts and methods. Participants' questionnaire responses showed that they became interested in science experiments and formed a strong connection between school knowledge and common knowledge.
\end{abstract}

Keywords: Common Knowledge, School Knowledge, Learning Science, Science Experiments.

\section{Introduction}

In this paper, we describe the development and evaluation of a mobile search system for science experiments in order to connect school knowledge to common knowledge. With the rapid development of mobile devices, the learning environment has extended beyond the school's computer room. Mobile learning environments are now being used in classrooms that do not have desktop PCs as well as even outside of the classroom. In the future, we need to discuss not only the wealth of learning resources made available by such environments but also how to use them.

Much of the knowledge in a learner's life comes from school; we refer to the knowledge taught in school as school knowledge. Knowledge becomes useful when the learner applies it in everyday life, but school knowledge is known not to be applied often in this way[1]. The knowledge used in everyday life is called common knowledge. To convert school knowledge to common knowledge, it is effective to apply 
school knowledge to everyday life[2]. It is important, therefore, to develop a system that provides learning resources and allows learners to apply school knowledge to real world situations. In our research, we constructed a database containing various experiments that teach certain scientific concepts and also developed a mobile search system that provides learners with experiments based on their present location when they $\log$ into the system.

\section{Mobile Search System for Science Experiments}

\subsection{System Structure}

Fig. 1 shows an overview of the learning activities our system provides. In the system, learners are required first to input their present location and then the system provides a list of possible experiments that takes into consideration the possible tools available at the learner's present location. The learners then select an experiment and conduct it. Finally, our system explains the meaning of the experiment so that they can apply various scientific concepts from the experiment to everyday life.

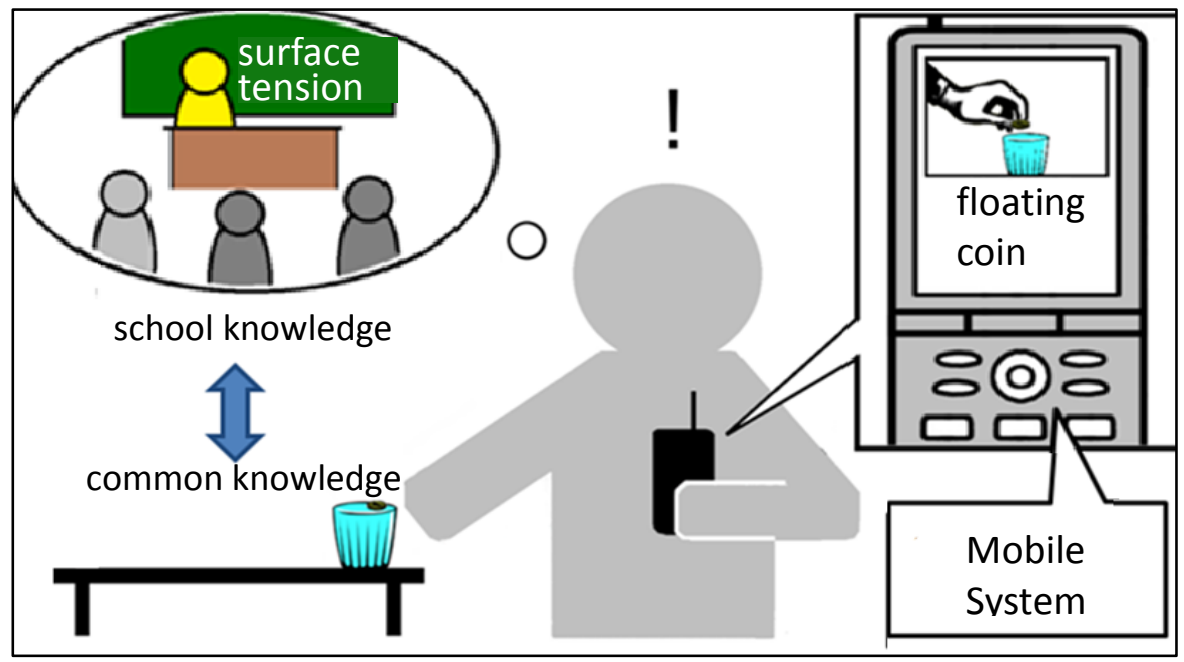

Fig. 1. Example of a Learning Activity in Our System

Fig. 2 shows the structure of our system, consisting of a server and a mobile device. The server program calculates the feasibility of an experiment using database values. The mobile system provides the required interface for searches and displays the experiments through the use of a search module. By inputting their present location, learners are shown a list of science experiments that can be conducted there; the list also shows the feasibility of the experiment with the tools necessary to complete it. The learners select and perform one of the experiments. Next, usage logs are sent to the server. 


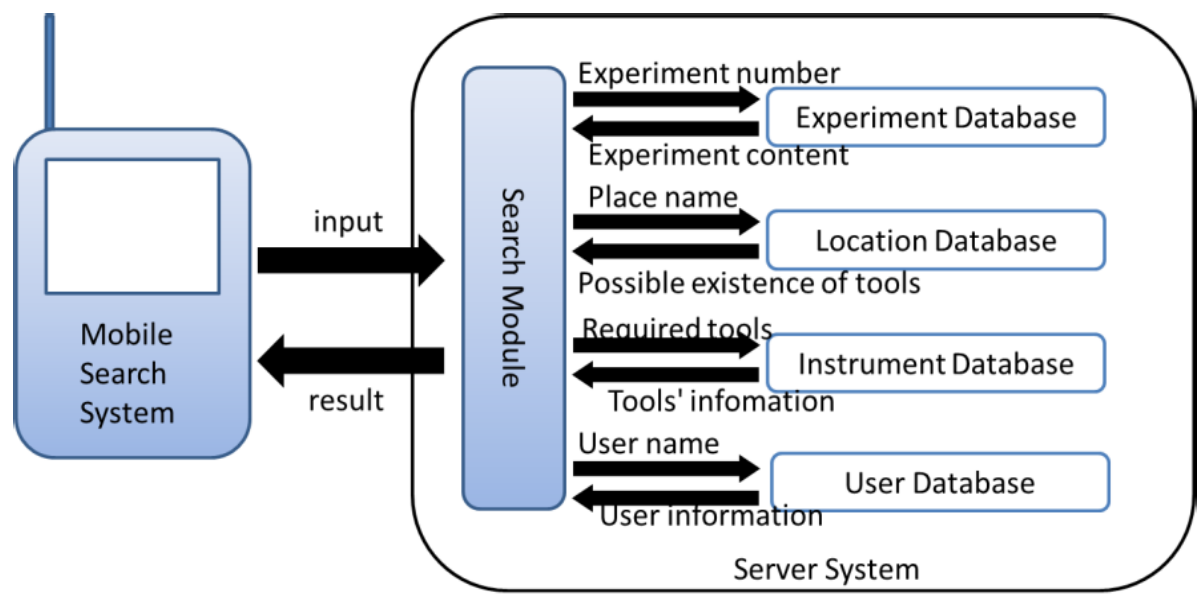

Fig. 2. The Structure of Our System

\subsection{Experiment Database}

The experiment database contains the experiment number as well as other information on content of the experiment. An example of the content information can be seen below. In the database, there are 61 experiments selected from a book published by Hans Jurgen Press [3].

\section{Experiment Number: 0}

2. Experiment Name: Floating coin

3. Tools: Water, coin, glass

4. Conditions: none

5. Experimental question:

What will happen when you put a coin in a glass filled with water?

\section{Steps:}

First, fill a glass with water.

Second, slowly place a coin on the surface of the water in the glass.

7. Result:

The coin floats in water.

8. Explanation:

A coin usually sinks because it is made of metal. The coin, however, floats when placed slowly on the water surface in a glass filled with water because the force of gravity of the coin and the combination of upper forces (which consist of surface tension and the force of buoyancy) are in balance.

9. Related scientific concepts:

Surface Tension, Force of Buoyancy, Equilibrium of Force

10. Related phenomena:

Pond skater floats in water. 


\subsection{Instrument Database}

The instrument database contains all the tools necessary to complete an experiment contained in the experiment database. The instrument database contains 105 tools in all.

\subsection{Location Database}

The location database contains the probability values of tools existing in 16 locations that learners visit in their everyday lives; we refer to this probability as "existence probability." In our system, the feasibility of an experiment is calculated based on the existence probability of the tools necessary for an experiment. The existence probability ranges from 0 to 1.0 .

The existence probability consists of two types of data: default data and individual data.

\section{The Default Existence Probability}

The default existence probability value is calculated through the results of a survey of 56 students who went to various locations and searched for tools. The calculation used is:

$$
D E P_{i k}=\frac{s}{56}
$$

$D E P_{i k}$ : Default existence probability of a tool $k$ in location $i$ $s$ : Number of students who can find the tool $k$ in location $i$

\section{The Individual Existence Probability}

In our system, the existence probability of tools is calculated on a per-user basis. If a user conducts an experiment, the individual existence probability for that given location is updated. The existence probability of the tools used in the experiment at the given location is updated to 1.0 because the tools are confirmed to be presently at the location. However, potential dilution of the tools increases with time, so the increased existence probability is decreased to the default existence probability over time. The calculation used is:

$$
\begin{gathered}
I E P_{i k}=a t+L I_{i k} \\
a=\frac{-1.0}{60 \text { days } * 24 \text { hours } * 60 \mathrm{~min} .}=\frac{-1.0}{8.64 * 10^{4}}
\end{gathered}
$$

$I E P_{i k}$ : Individual existence probability of a tool $k$ in location $i$

$L I_{i k}$ : Last login time associated with the individual existence probability of tool $k$ in location $i$

$t$ : Time after last login 


\subsection{User Database}

The user database contains each user's name, login time, and the number of experiments the user has conducted with the system.

\subsection{Search Module}

When learners select their present location, our search module determines the feasibility of the various experiments using the databases. The feasibility values are calculated based on the existence probabilities of the necessary tools for the experiment. The calculation is:

$$
\begin{gathered}
F E_{i j}=\prod_{k=1}^{n_{j}} E P_{i k} \\
E P_{i k}=\max \left(D E P_{i k}, I E P_{i k}\right)
\end{gathered}
$$

$F E_{i j}$ : Feasibility of the experiment $j$ in location $i$

$E P_{i k}$ : Existence probability of a tool $k$ in location $i$

$n_{j}$ : Number of required tools for experiment $j$

Our system calculates all $F E_{i j}$ values for a selected location $i$ and arranges the experiments in descending order of feasibility with the search module.

\section{$2.7 \quad$ Interface}

Fig. 3 shows the location selection interfaces. Learners are first required to select their present location.
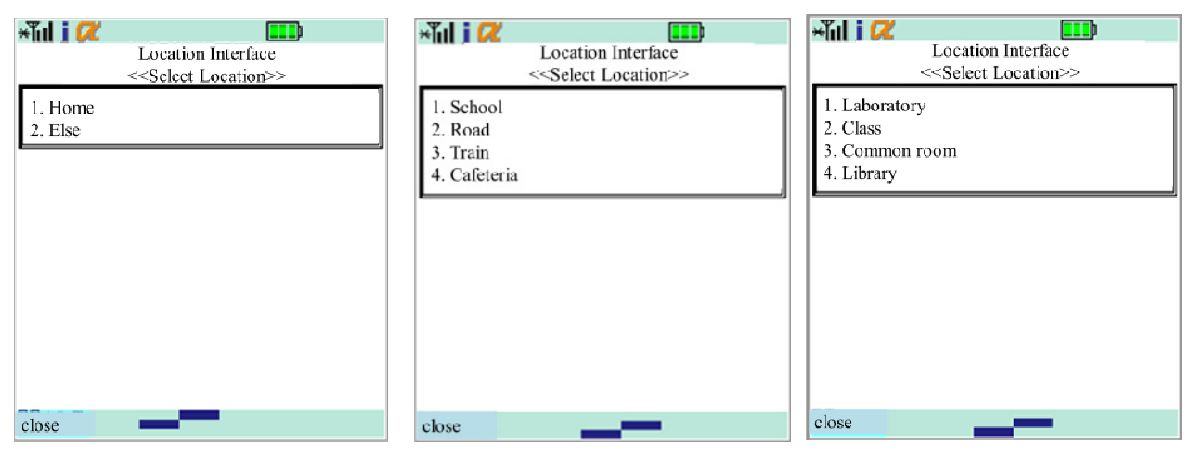

Fig. 3. Location Selection Interfaces

Next, the system calculates the feasibility of each experiment for the selected location $\left(F E_{i j}\right)$ and displays experiments in descending order of feasibility (Fig. 4). Learners then select an experiment that they are willing and able to conduct. 


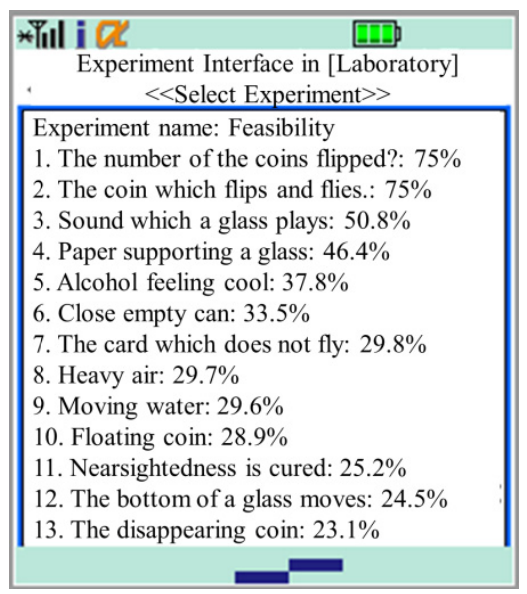

Fig. 4. Experiment Selection Interface

After selecting an experiment, the system shows the learners the experimental question and the tools necessary to complete the selected experiment, as seen on the left side of Fig. 5. When the learners select an experiment, they can check the experimental steps, as seen on the right side of Fig. 5. Finally, they conduct the experiment, as seen in Fig. 6, and check the experimental result and explanation in the system.
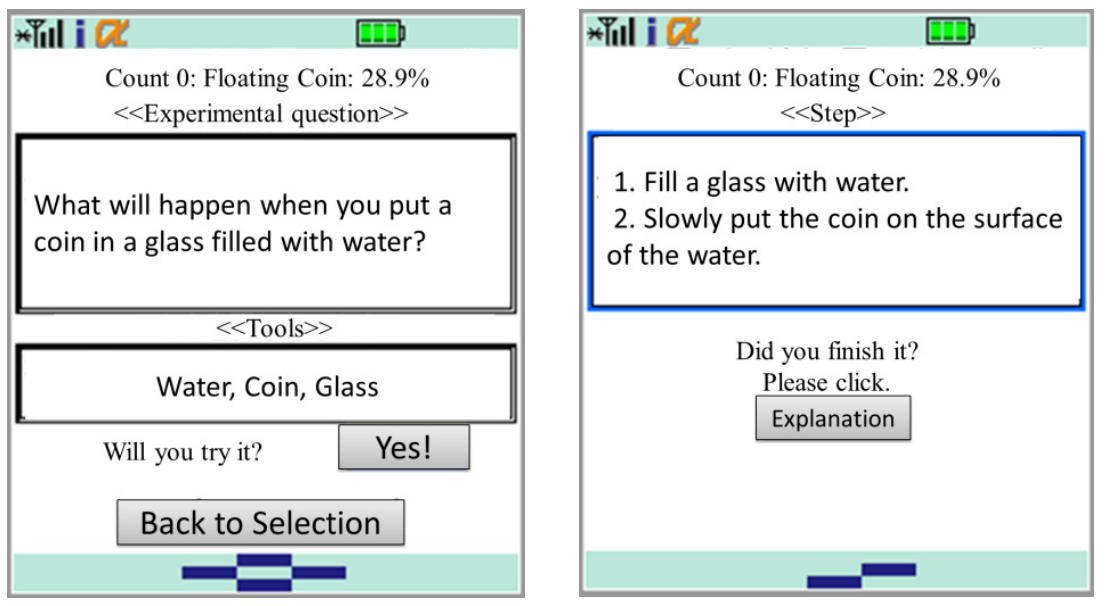

Fig. 5. Experimental question and Tools Interface(left), and Step Interface (right) 


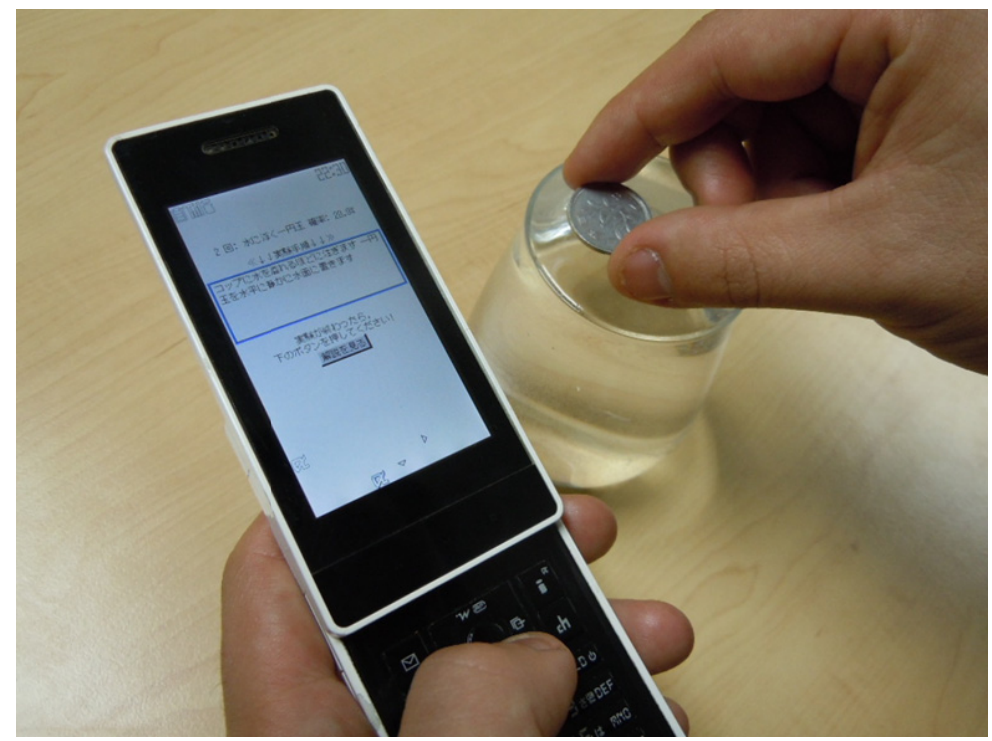

Fig. 6. Performing an Experiment

\section{Evaluation Experiments}

We report here the results of two experiments (Experiment 1 and Experiment 2). The objective of Experiment 1 was to evaluate the feasibility calculation. In our mobile system, experiments are provided based on feasibility for a given location. The objective Experiment 2 was to evaluate the effectiveness of the system for enabling learners to connect their school knowledge to their common knowledge.

\subsection{Experiment 1}

In Experiment 1, we evaluated whether the system could provide feasible experiments for various locations.

There were 7 test participants: 6 university students and 1 graduates. The participants tried 10 times to perform the provided experiments in 4 locations around the university (labs, classrooms, cafeteria, and library). Each location was assigned to 2 people, thus 20 evaluations were conducted per location.

We evaluated the experiment feasibility results on a precision basis. Precision was calculated by dividing the number of actual conducted experiments by the number of experiments proposed by our system. The precision results from the laboratory, classroom, cafeteria, and library were $0.9,0.2,0.7$, and 0.2 , respectively. The precision results from the classroom and library were lower than for the laboratory and cafeteria because the classroom and the library do not have as many tools available for experiments. 


\subsection{Experiment 2}

\section{Objective}

To evaluate if the system was effective for connecting school knowledge and common knowledge

\section{Subjects}

Seven university students and five graduates

\section{Procedure}

1. Pre-test (15 min.)

2. Explanation of how to use our system.

3. Use of our system (1 week)

4. Post-test (15 min.)

5. Questionnaire (10 min.)

\section{Contents of the Test}

Fig. 7 shows the content of the pre-test and post-test. In the tests, learners are required to describe the experiments that can be conducted in their everyday life. We marked when they described appropriate places, tools, concepts, and methods.

\section{Test (15 min.)}

Your Name(

)

Please describe as many physics experiments that can be conducted at a certain location as possible. Moreover, please describe the required tools, the related concepts, and the experimental procedure.

Example:

Location

My room

Required tools ( Lid of a box, marble

Related concepts ( Law of conservation of energy

)

Procedure

Ten glass balls are arranged in one row along the bottom edge of the inside of a box. One ball is strongly thrown at the end of the line of balls. As a result, one ball moves out of the opposite end.

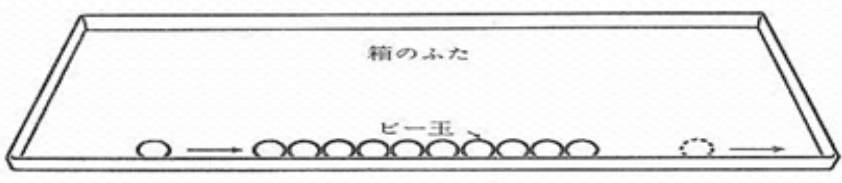

Fig. 7. Content of the Pre-test and Post-test 


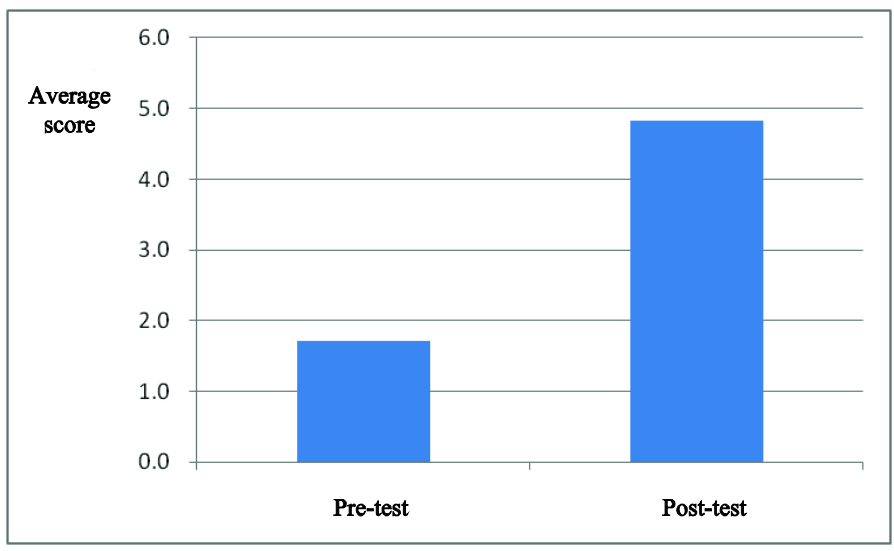

Fig. 8. Average Score of the Pre-test and Post-test

Fig. 8 shows the results of the pre-test and post-test, with an average score of 1.7 for the former and 4.8 for the latter. The statistical difference was determined by paired $t$ test. Table 1 shows that the result of the $t$ test. The average score of post-test was significantly higher $(\mathrm{p}<0.01)$ than pre-test.

Table 1. The result of $t$ test

\begin{tabular}{|c|c|c|}
\hline $\begin{array}{c}\text { degree of free- } \\
\text { dom }\end{array}$ & t value & $\mathrm{p}$ value \\
\hline 11 & 2.2 & $4.0^{*} 10^{-4}$ \\
\hline
\end{tabular}

The participants answered a questionnaire on their thoughts about the system. The participants were asked whether they minded applying school knowledge to their everyday life, to which they responded using a 5-point numerical Likert scale. We found that favorable results: the average score of the item "Did you get more interested in the science experiment?" was 4.42, that for the item "Have you improved your understanding of school knowledge?" was 4.75, and that for the item "Did you start to consider school knowledge in everyday life?" was 4.25 .

\section{Conclusion}

In this paper, we proposed a method to enable learners to use their school knowledge in everyday life. We developed and evaluated our mobile search system for learners to complete science experiments in their everyday lives, finding that they were able to connect school knowledge and common knowledge.

In the evaluation experiment, 15 participants used our system over the course of 1 week. The results indicated that they became able to consider appropriate experiments, describe appropriate locations, and understand scientific concepts and 
methods. The questionnaire results revealed that they became interested in science experiments and considered school knowledge to be highly applicable to everyday life and thus to common knowledge.

\section{References}

1. John, T., Bruer, T.J.: Schools for thought: a science of learning in the classroom. MIT Press (1993)

2. Tomoto, T., Horiguchi, T., Hirashima, T., Takeuchi, A.: Virtual Experimental Environment to Support Learning by Physical Experiment Designing. The Journal of Information and Systems in Education 8(1), 72-83 (2009)

3. Jurgen, H.: Press: SPIEL-DAS WISSEN SCHAFFT, Ravensburger Buchverlag; Neuauflage $(20040$ 